\title{
Quantum calcium-ion interactions with EEG
}

\author{
Lester Ingber \\ Lester Ingber Research \\ Ashland, Oregon 97520 \\ ingber@alumni.caltech.edu [https://www.ingber.com]
}

\begin{abstract}
Background: Previous papers have developed a statistical mechanics of neocortical interactions (SMNI) fit to short-term memory and EEG data. Adaptive Simulated Annealing (ASA) has been developed to perform fits to such nonlinear stochastic systems. An N-dimensional path-integral algorithm for quantum systems, qPATHINT, has been developed from classical PATHINT. Both fold short-time propagators (distributions or wave functions) over long times. Previous papers applied qPATHINT to two systems, in neocortical interactions and financial options. Objective: In this paper the quantum path-integral for Calcium ions is used to derive a closed-form analytic solution at arbitrary time that is used to calculate interactions with classical-physics SMNI interactions among scales. Using fits of this SMNI model to EEG data, including these effects, will help determine if this is a reasonable approach. Method: Methods of mathematical-physics for optimization and for path integrals in classical and quantum spaces are used for this project. Studies using supercomputer resources tested various dimensions for their scaling limits. In this paper the quantum path-integral is used to derive a closed-form analytic solution at arbitrary time that is used to calculate interactions with classical-physics SMNI interactions among scales. Results: The mathematical-physics and computer parts of the study are successful, in that there is modest improvement of cost/objective functions used to fit EEG data using these models. Conclusion: This project points to directions for more detailed calculations using more EEG data and qPATHINT at each time slice to propagate quantum calcium waves, synchronized with PATHINT propagation of classical SMNI.
\end{abstract}

Key words: quantum mechanics; EEG; short term memory; astrocytes; neocortical dynamics; vector potential

\section{Introduction}

This project calculates quantum $\mathrm{Ca}^{2+}$ interactions with EEG. In this paper, EEG is synonymous with large-scale neocortical firings during attentional tasks as measured by large-amplitude electroencephalographic (EEG) recordings. In this paper, only very specific calcium ions, $\mathrm{Ca}^{2+}$, are considered, those arising from regenerative calcium waves generated at tripartite neuron-astrocyteneuron synapses. Indeed, it is important to note that $\mathrm{Ca}^{2+}$ ions, and specifically $\mathrm{Ca}^{2+}$ waves, influence many processes in the brain, but this study focuses on free waves generated at tripartite synapses because of their calculated direct interactions with large synchronous neuronal firings.

Section 2 reviews the background of the main model used, Statistical Mechanics of Neocortical Interactions (SMNI).

Section 3 reviews the code Adaptive Simulated Annealing (ASA), used for optimization of many systems - fitting models to real data, e.g., fits to EEG data reported here.

Section 4 reviews the development of path-integral codes, PATHINT and qPATHINT, used for propagation of conditional probabilities and quantum-mechanical wave-functions, as reported here. 
Section 5 gives new results of inclusion of quantum-mechanical interactions of $\mathrm{Ca}^{2+}$ wave-packets with EEG.

Section 6 reviews some applications of this project.

Section 7 gives the conclusion.

The theory and codes for ASA and [q]PATHINT have been well tested across many disciplines by multiple users. This particular project most certainly is speculative, but it is testable. As reported here, fitting such models to EEG tests some aspects of this project. This is a somewhat indirect path, but not novel to many physics paradigms that are tested by experiment or computation. A detailed future path is described in the [q]PATHINT review Section.

While SMNI has been developed since 1981, and been confirmed by many tests, this evolving model including ionic scales has been part of multiple papers relatively recently, since 2012. Classical physics calculations support these extended SMNI models and are consistent with experimental data. Quantum physics calculations also support these extended SMNI models and, while they too are consistent with experimental data, it is quite speculative that they can persist in neocortex. Admittedly, it is surprising that detailed calculations continue to support this model, and so it is worth continued examination it until it is theoretically or experimentally proven to be false.

\section{Statistical Mechanics of Neocortical Interactions (SMNI)}

SMNI has been developed since 1981, scaling aggregate synaptic interactions to neuronal firings, up to minicolumnar-macrocolumnar columns of neurons to mesocolumnar dynamics, up to columns of neuronal firings, up to regional macroscopic sites (Ingber, 1981, 1982, 1983, 1984, 1985a, 1994).

SMNI has calculated agreement/fits with experimental data from various aspects of neocortical interactions, e.g., properties of short-term memory (STM) (Ingber, 2012a), including its capacity (auditory $7 \pm 2$ and visual $4 \pm 2$ ) (Ericsson and Chase, 1982; Zhang and Simon, 1985), duration, stability, primacy versus recency rule, as well other phenomenon, e.g., Hick's law (Hick, 1952; Ingber, 1999; Jensen, 1987), interactions within macrocolumns calculating mental rotation of images, etc (Ingber, 1982, 1983, 1984, 1985a, 1994). SMNI scaled mesocolumns across neocortical regions to fit EEG data (Ingber, 1997a b, 2012a). Fig. 1 depicts this model (Ingber, 1983). 


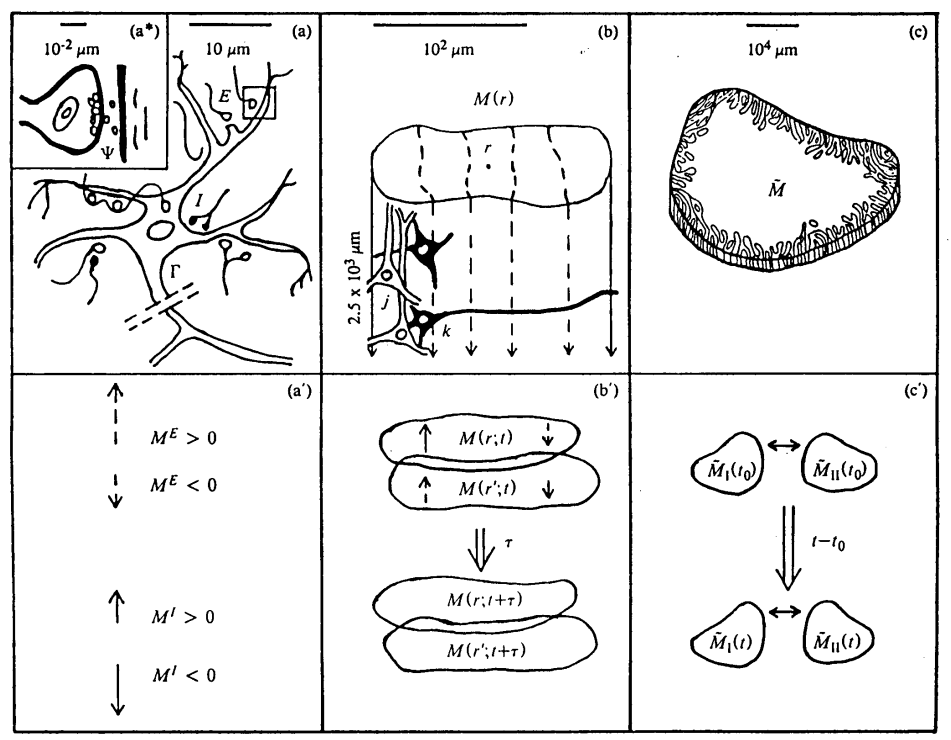

Figure 1 illustrates three SMNI biophysical scales (Ingber, 1982, 1983): (a)-(a*)-(a') microscopic neurons; (b)-(b') mesocolumnar domains; (c)-(c') macroscopic regions.

$\left(\mathrm{a}^{*}\right)$ : synaptic inter-neuronal interactions, scaled up to mesocolumns, phenomenologically described by the mean and variance of a distribution $\Psi$ (a): intraneuronal transmissions phenomenologically described by the mean and variance of $\Gamma$ (a'): collective mesocolumnar-averaged inhibitory $(I)$ and excitatory $(E)$ neuronal firings $M$

(b): vertical organization of minicolumns including their horizontal layers, yielding a physiological entity, the mesocolumn (b'): overlapping mesocolumns at locations $r$ and $r^{\prime}$ from times $t$ and $t+\tau, \tau$ on the order of $10 \mathrm{msec}$

(c): macroscopic regions of neocortex arising from many mesocolumnar domains $\left(c^{\prime}\right)$ : regions coupled by long-ranged interactions

\subsection{Synaptic Interactions}

The short-time conditional probability distribution of firing of a given neuron firing given justprevious firings of other neurons is calculated from chemical and electrical intra-neuronal interactions (Ingber, 1982, 1983). Given its previous interactions with $k$ neurons within $\tau_{j}$ of 5-10 msec, the conditional probability that neuron $j$ fires $\left(\sigma_{j}=+1\right)$ or does not fire $\left(\sigma_{j}=-1\right)$ is

$$
\begin{gathered}
p_{\sigma_{j}}=\Gamma \Psi=\frac{\exp \left(-\sigma_{j} F_{j}\right)}{\exp \left(F_{j}\right)+\exp \left(-F_{j}\right)} \\
F_{j}=\frac{V_{j}-\sum_{k} a_{j k}^{*} v_{j k}}{\left(\pi \sum_{k^{\prime}} a_{j k^{\prime}}^{*}\left(v_{j k^{\prime}}^{2}+\phi_{j k^{\prime}}^{2}\right)\right)^{1 / 2}} \\
a_{j k}=\frac{1}{2} A_{|j k|}\left(\sigma_{k}+1\right)+B_{j k}
\end{gathered}
$$

The contribution to polarization achieved at an axon given activity at a synapse, taking into account averaging over different neurons, geometries, etc., is given by $\Gamma$, the "intra-neuronal" probability distribution. $\Psi$ is the "inter-neuronal" probability distribution, of thousands of quanta of neurotransmitters released at one neuron's presynaptic site effecting a (hyper-)polarization at 
another neuron's postsynaptic site, taking into account interactions with neuromodulators, etc. This development holds for $\Gamma$ Poisson, and for $\Psi$ Poisson or Gaussian.

$V_{j}$ is the depolarization threshold in the somatic-axonal region. $v_{j k}$ is the induced synaptic polarization of $E$ or $I$ type at the axon, and $\phi_{j k}$ is its variance. The efficacy $a_{j k}$ is a sum of $A_{j k}$ from the connectivity between neurons, activated if the impinging $k$-neuron fires, and $B_{j k}$ from spontaneous background noise. The efficacy is related to the impedance across synaptic gaps.

\section{$2.2 \quad$ Neuronal Interactions}

Aggregation up to the mesoscopic scale from the microscopic synaptic scale uses mesoscopic probability $P$

$$
\begin{gathered}
P=\prod_{G} P^{G}\left[M^{G}(r ; t+\tau) \mid M^{\bar{G}}\left(r^{\prime} ; t\right)\right] \\
=\sum_{\sigma_{j}} \delta\left(\sum_{j E} \sigma_{j}-M^{E}(r ; t+\tau)\right) \delta\left(\sum_{j I} \sigma_{j}-M^{I}(r ; t+\tau)\right) \prod_{j}^{N} p_{\sigma_{j}}
\end{gathered}
$$

$M$ represents a mesoscopic scale of columns of $N$ neurons, with subsets $E$ and $I$, represented by $p_{q_{i}}$. The "delta"-functions $\delta$-constraint represents an aggregate of many neurons in a column. $G$ is used to represent excitatory $(E)$ and inhibitory $(I)$ contributions. $\bar{G}$ designates contributions from both $E$ and $I$.

The path integral is derived in terms of mesoscopic Lagrangian $L$. The short-time distribution of firings in a minicolumn, given its just previous interactions with all other neurons in its macrocolumn, is thereby defined.

\subsection{Columnar Interactions}

In the prepoint (Ito) representation the SMNI Lagrangian $L$ is

$$
\begin{gathered}
L=\sum_{G, G^{\prime}}(2 N)^{-1}\left(\dot{M}^{G}-g^{G}\right) g_{G G^{\prime}}\left(\dot{M}^{G^{\prime}}-g^{G^{\prime}}\right) /(2 N \tau)-V^{\prime} \\
g^{G}=-\tau^{-1}\left(M^{G}+N^{G} \tanh F^{G}\right) \\
g^{G G^{\prime}}=\left(g_{G G^{\prime}}\right)^{-1}=\delta_{G}^{G^{\prime}} \tau^{-1} N^{G} \operatorname{sech}^{2} F^{G} \\
g=\operatorname{det}\left(g_{G G^{\prime}}\right)
\end{gathered}
$$

The threshold factor $F^{G}$ is derived as

$$
\begin{gathered}
F^{G}=\sum_{G^{\prime}} \frac{\nu^{G}+\nu^{\ddagger E^{\prime}}}{\left((\pi / 2)\left[\left(v_{G^{\prime}}^{G}\right)^{2}+\left(\phi_{G^{\prime}}^{G}\right)^{2}\right]\left(\delta^{G}+\delta^{\ddagger E^{\prime}}\right)\right)^{1 / 2}} \\
\nu^{G}=V^{G}-a_{G^{\prime}}^{G} v_{G^{\prime}}^{G} N^{G^{\prime}}-\frac{1}{2} A_{G^{\prime}}^{G} v_{G^{\prime}}^{G} M^{G^{\prime}}, \nu^{\ddagger E^{\prime}}=-a_{E^{\prime}}^{\ddagger E} v_{E^{\prime}}^{E} N^{\ddagger E^{\prime}}-\frac{1}{2} A_{E^{\prime}}^{\ddagger E} v_{E^{\prime}}^{E} M^{\ddagger E^{\prime}} \\
\delta^{G}=a_{G^{\prime}}^{G} N^{G^{\prime}}+\frac{1}{2} A_{G^{\prime}}^{G} M^{G^{\prime}}, \delta^{\ddagger E^{\prime}}=a_{E^{\prime}}^{\ddagger E} N^{\ddagger E^{\prime}}+\frac{1}{2} A_{E^{\prime}}^{\ddagger E} M^{\ddagger E^{\prime}}
\end{gathered}
$$




$$
a_{G^{\prime}}^{G}=\frac{1}{2} A_{G^{\prime}}^{G}+B_{G^{\prime}}^{G}, a_{E^{\prime}}^{\ddagger E}=\frac{1}{2} A_{E^{\prime}}^{\ddagger E}+B_{E^{\prime}}^{\ddagger E}
$$

where $A_{G^{\prime}}^{G}$ is the columnar-averaged direct synaptic efficacy, $B_{G^{\prime}}^{G}$ is the columnar-averaged backgroundnoise contribution to synaptic efficacy. The “ ” parameters arise from regional interactions across many macrocolumns.

\subsection{SMNI Parameters From Experiments}

All values of parameters and their bounds are taken from experimental data, not arbitrarily fit to specific phenomena.

$N^{G}=\left\{N^{E}=160, N^{I}=60\right\}$ was set for for visual neocortex, $\left\{N^{E}=80, N^{I}=30\right\}$ was set for all other neocortical regions, $M^{G^{\prime}}$ and $N^{G^{\prime}}$ in $F^{G}$ are afferent macrocolumnar firings scaled to efferent minicolumnar firings by $N / N^{*} \approx 10^{-3}$. $N^{*}$ is the number of neurons in a macrocolumn, about $10^{5}$. $V^{\prime}$ includes nearest-neighbor mesocolumnar interactions. $\tau$ is usually considered to be on the order of $5-10 \mathrm{~ms}$.

Other values also are consistent with experimental data, e.g., $V^{G}=10 \mathrm{mV}, v_{G^{\prime}}^{G}=0.1 \mathrm{mV}$, $\phi_{G^{\prime}}^{G}=0.03^{1 / 2} \mathrm{mV}$.

Nearest-neighbor interactions among columns give dispersion relations that were used to calculate speeds of mental visual rotation (Ingber, 1982, 1983).

The wave equation cited by EEG theorists, permitting fits of SMNI to EEG data (Ingber, 1995a), was derived using the variational principle applied to the SMNI Lagrangian.

This creates an audit trail from synaptic parameters to the statistically averaged regional Lagrangian.

\subsection{Previous Applications}

\subsubsection{Verification of basic SMNI Hypothesis}

The core SMNI hypothesis first developed circa 1980 (Ingber, 1981, 1982, 1983) is that highly synchronous patterns of neuronal firings in fact process high-level information. Only since 2012 has this hypothesis been verified experimentally (Asher, 2012; Salazar et al., 2012).

\subsubsection{SMNI Calculations of Short-Term Memory (STM)}

SMNI calculations agree with observations (Ingber, 1982, 1983, 1984, 1985a, 1994, 1995b, 1997a, 1999, 2011, 2012a b, 2015, 2016a, 2017a b; Ingber et al., 2014, Nunez et al., 2013): This list includes:

capacity (auditory $7 \pm 2$ and visual $4 \pm 2$ ) (Ingber, 1984)

duration (Ingber, 1985a)

stability (Ingber, 1985a)

primacy versus recency rule (Ingber, 1985a b)

Hick's law (reaction time and $g$ factor) (Ingber, 1999)

nearest-neighbor minicolumnar interactions $=>$ mental rotation of images (Ingber, 1982, 1983)

derivation of basis for EEG (Ingber, 1985c, 1995a) 


\subsubsection{Three Basic SMNI Models}

Three basic models were developed by slightly changing the background firing component of the columnar-averaged efficacies $B_{G^{\prime}}^{G}$ within experimental ranges, which modify $F^{G}$ threshold factors to yield in the conditional probability:

(a) case EC, dominant excitation subsequent firings

(b) case IC, inhibitory subsequent firings

(c) case BC, balanced between EC and IC

This is consistent with experimental evidence of shifts in background synaptic activity under conditions of selective attention (Briggs et al., 2013, Mountcastle et al., 1981), This enables a Centering Mechanism (CM) on case $\mathrm{BC}$, giving $\mathrm{BC}^{\prime}$, wherein the numerator of $F^{G}$ only has terms proportional to $M^{E^{\prime}}, M^{I^{\prime}}$ and $M^{\ddagger E^{\prime}}$, i.e., zeroing other constant terms by resetting the background parameters $B_{G^{\prime}}^{G}$, still within experimental ranges. This brings in a maximum number of minima into the physical firing $M^{G}$-space, due to the minima of the new numerator in being in a parabolic trough defined by

$$
A_{E}^{E} M^{E}-A_{I}^{E} M^{I}=0
$$

about which nonlinearities develop multiple minima identified with STM phenomena.

In current projects a Dynamic CM (DCM) model is used, resetting $B_{G^{\prime}}^{G}$ every few epochs of $\tau$. Such changes in background synaptic activity on such time scales are seen during attentional tasks (Briggs et al., 2013).

\subsection{Comparing EEG Testing Data with Training Data}

Using EEG data from http://physionet.nlm.nih.gov/pn4/erpbci (Citi et al., 2010, Goldberger et al., 2000), SMNI was fit to highly synchronous waves (P300) during attentional tasks, for each of 12 subjects, it was possible to find 10 Training runs and 10 Testing runs (Ingber, 2016a).

Spline-Laplacian transformations on the EEG potential $\Phi$ are proportional to the SMNI $M^{G}$ firing variables at each electrode site. The electric potential $\Phi$ is experimentally measured by EEG, not $\mathbf{A}$, but both are due to the same currents $\mathbf{I}$. Therefore, $\mathbf{A}$ is linearly proportional to $\Phi$ with a simple scaling factor included as a parameter in fits to data. Additional parameterization of background synaptic parameters, $B_{G^{\prime}}^{G}$ and $B_{E^{\prime}}^{\ddagger E}$, modify previous work.

The $\mathbf{A}$ model outperformed the no- $\mathbf{A}$ model, where the no-A model simply has used $\mathbf{A}$-nondependent synaptic parameters. Cost functions with an $|\mathbf{A}|$ model were much worse than either the A model or the no-A model. Runs with different signs on the drift and on the absolute value of the drift also gave much higher cost functions than the $\mathbf{A}$ model.

\subsection{STM PATHINT Calculations}

\subsubsection{PATHINT STM}

The evolution of a Balanced Centered model (BC) after 500 foldings of $\Delta t=0.01,5$ unit of relaxation time $\tau$, exhibits the existence of ten well developed peaks. These peaks are identified with possible trappings of firing patterns.

This describes the " $7 \pm 2$ " rule, as calculated by SMNI PATHINT in Fig. 2 (Ingber and Nunez 1995). 


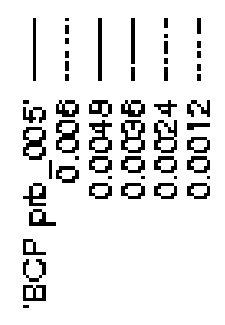

․

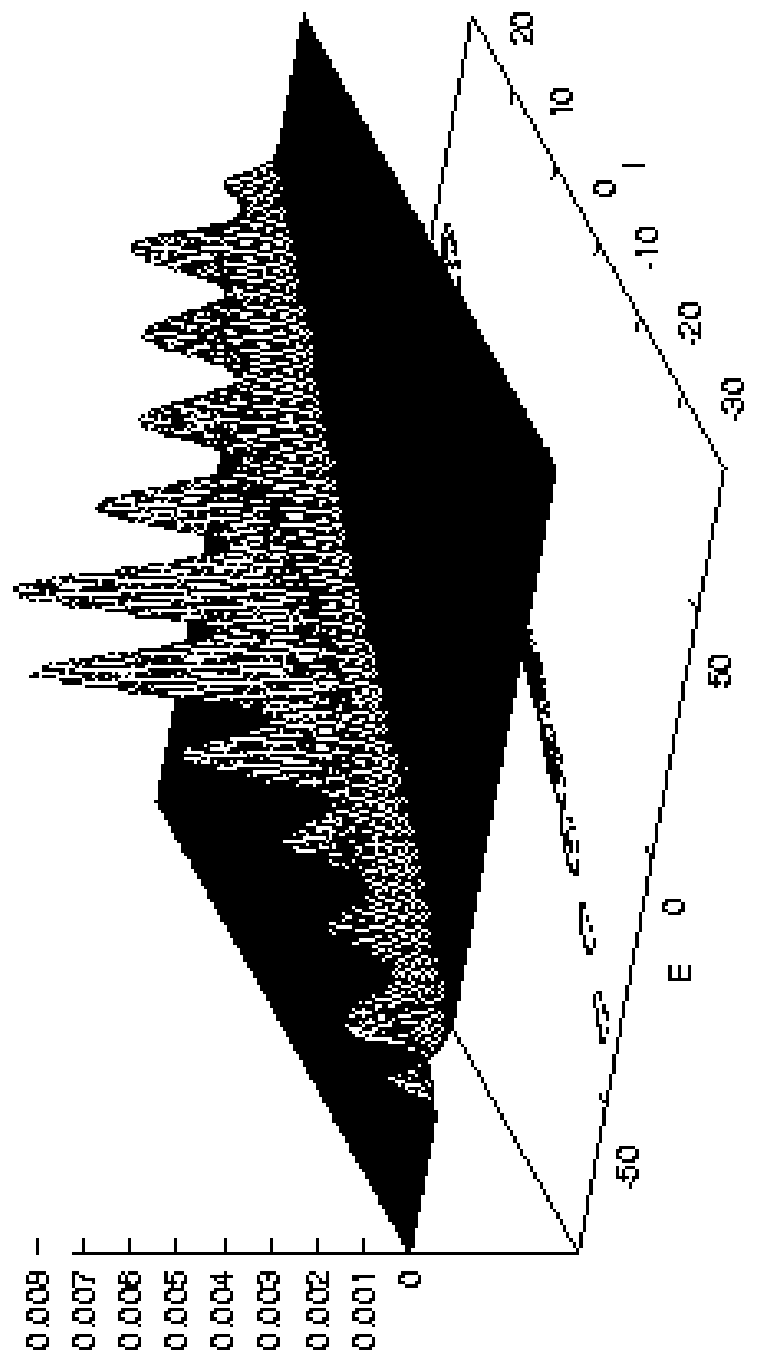

Figure 2 illustrates SMNI STM Model BC at the evolution at $5 \tau$ (Ingber and Nunez, 1995).

\subsubsection{PATHINT STM Visual}

The evolution of a Balanced Centered Visual model (BCV) after 1000 foldings of $\Delta t=0.01,10$ unit of relaxation time $\tau$, exhibits the existence of four well developed peaks. These peaks are identified with possible trappings of firing patterns. Other peaks at lower scales are clearly present, numbering on the same order as in the BC' model, as the strength in the original peaks dissipates throughout firing space, but these are much smaller and therefore much less probable to be accessed.

This describes the " $4 \pm 2$ " rule for visual STM, as calculated by SMNI PATHINT in Fig. 3 (Ingber and Nunez, 1995). 




Figure 3 illustrates SMNI STM Model BCV at the evolution at 10 $\tau$ (Ingber and Nunez, 1995).

\subsection{Tripartite Synaptic Interactions}

The human brain contains over $10^{11}$ cells, about half of which are neurons. The other half are glial cells. Astrocytes comprise a good fraction of glial cells, possibly the majority. Many papers examine the influence of astrocytes on synaptic processes (Agulhon et al., 2008; Araque and Navarrete, 2010, Banaclocha et al., 2010; Bellinger, 2005, Innocenti et al., 2000; A. Pereira and Furlan, 2009; Reyes and Parpura, 2009; Scemes and Giaume, 2006; Volterra et al., 2014).

http://www.astrocyte.info claims they are the most numerous cells in the human brain. Unlike the previous ideology of astrocytes being "filler" cells, they are very active in the central nervous system and greatly outnumber neurons,

Glutamate release from astrocytes through a $\mathrm{Ca}^{2+}$-dependent mechanism can activate receptors 
at the presynaptic terminals. Intercellular calcium waves (ICWs) may travel over hundreds of astrocytes propagating over many neuronal synapses. ICWs contribute to control synaptic activity. Glutamate is released in a regenerative manner, with subsequent cells that are involved in the calcium wave releasing additional glutamate (Ross, 2012).

$\left[\mathrm{Ca}^{2+}\right]$ (concentrations of $\mathrm{Ca}^{2+}$ ) affect increased release probabilities at synaptic sites, by enhancing the release of gliotransmitters. (Free $\mathrm{Ca}^{2+}$ waves are considered here, not intracellular astrocyte calcium waves in situ which also increase neuronal firings.)

These free regenerative $\mathrm{Ca}^{2+}$ waves, arising from astrocyte-neuron interactions, couple to the magnetic vector potential A produced by highly synchronous collective firings, e.g., during selective attention tasks, as measured by EEG.

\subsubsection{Canonical Momentum $\boldsymbol{\Pi}=\mathbf{p}+q \mathbf{A}$}

As derived in the Feynman (midpoint) representation of the path integral, the canonical momentum, $\boldsymbol{\Pi}$, defines the dynamics of a moving particle with momentum $\mathbf{p}$ in an electromagnetic field. In SI units,

$$
\mathbf{\Pi}=\mathbf{p}+q \mathbf{A}
$$

where $q=-2 e$ for $\mathrm{Ca}^{2+}, e$ is the magnitude of the charge of an electron $=1.6 \times 10^{-19} \mathrm{C}$ (Coulomb), and $\mathbf{A}$ is the electromagnetic vector potential. (In Gaussian units $\boldsymbol{\Pi}=\mathbf{p}+q \mathbf{A} / c$, where $c$ is the speed of light.) A represents three components of a 4 -vector.

\subsubsection{Vector Potential of Wire}

A columnar firing state is modeled as a wire/neuron with current $\mathbf{I}$ measured in $\mathrm{A}=$ Amperes $=$ $\mathrm{C} / \mathrm{s}$,

$$
\mathbf{A}(t)=\frac{\mu}{4 \pi} \int \frac{d r}{r} \mathbf{I}
$$

along a length $z$ observed from a perpendicular distance $r$ from a line of thickness $r_{0}$. If far-field retardation effects are neglected, this yields

$$
\mathbf{A}=\frac{\mu}{4 \pi} \mathbf{I} \log \left(\frac{r}{r_{0}}\right)
$$

where $\mu$ is the magnetic permeability in vacuum $=4 \pi 10^{-7} \mathrm{H} / \mathrm{m}$ (Henry/meter). Note the insensitive $\log$ dependence on distance.

The contribution to $\mathbf{A}$ includes minicolumnar lines of current from hundreds to thousands of macrocolumns, within a region not so large to include many convolutions, but still contributing to large synchronous bursts of EEG.

Electric $\mathbf{E}$ and magnetic $\mathbf{B}$ fields, derivatives of $\mathbf{A}$ with respect to $r$, do not possess this $\log -$ arithmic insensitivity to distance, and therefore they do not linearly accumulate strength within and across macrocolumns.

Estimates of contributions from synchronous firings to P300 measured on the scalp are tens of thousands of macrocolumns spanning 100 to 100's of $\mathrm{cm}^{2}$. Electric fields generated from a minicolumn may fall by half within 5-10 mm, the range of several macrocolumns.

There are other possible sources of magnetic vector potentials not described as wires with currents (Majhi and Ghosh, 2018). Their net effects plausibly would be included the vector magnetic potential of net synchrous firings, but not their functional forms as derived here. 


\subsubsection{Effects of Vector Potential on Momenta}

The momentum $\mathbf{p}$ for a $\mathrm{Ca}^{2+}$ ion with mass $m=6.6 \times 10^{-26} \mathrm{~kg}$, speed on the order of $50 \mu \mathrm{m} / \mathrm{s}$ to $100 \mu \mathrm{m} / \mathrm{s}$, is on the order of $10^{-30} \mathrm{~kg}-\mathrm{m} / \mathrm{s}$. Molar concentrations of $\mathrm{Ca}^{2+}$ waves, comprised of tens of thousands of free ions representing about $1 \%$ of a released set, most being buffered, are within a range of about $100 \mu \mathrm{m}$ to as much as $250 \mu \mathrm{m}$, with a duration of more than $500 \mathrm{~ms}$, and with $\left[\mathrm{Ca}^{2+}\right]$ ranging from $0.1-5 \mu \mathrm{M}\left(\mu \mathrm{M}=10^{-3} \mathrm{~mol} / \mathrm{m}^{3}\right)$.

The magnitude of the current is taken from experimental data on dipole moments $\mathbf{Q}=|\mathbf{I}| \hat{\mathbf{z}}$ where $\hat{\mathbf{z}}$ is the direction of the current $\mathbf{I}$ with the dipole spread over $z$. $\mathbf{Q}$ ranges from $1 \mathrm{pA}-\mathrm{m}=$ $10^{-12} \mathrm{~A}-\mathrm{m}$ for a pyramidal neuron (Murakami and Okada, 2006), to $10^{-9} \mathrm{~A}-\mathrm{m}$ for larger neocortical mass (Nunez and Srinivasan, 2006). These currents give rise to $q \mathbf{A} \approx 10^{-28} \mathrm{~kg}-\mathrm{m} / \mathrm{s}$. The velocity of a $\mathrm{Ca}^{2+}$ wave can be $\approx 20-50 \mu \mathrm{m} / \mathrm{s}$. In neocortex, a typical $\mathrm{Ca}^{2+}$ wave of 1000 ions, with total mass $m=6.655 \times 10^{-23} \mathrm{~kg}$ times a speed of $\approx 20-50 \mu \mathrm{m} / \mathrm{s}$, gives $\mathbf{p} \approx 10^{-27} \mathrm{~kg}-\mathrm{m} / \mathrm{s}$.

Taking $10^{4}$ synchronous firings in a macrocolumn, leads to a dipole moment $|\mathbf{Q}|=10^{-8} \mathrm{~A}-\mathrm{m}$. Taking $z$ to be $10^{2} \mu \mathrm{m}=10^{-4} \mathrm{~m}$, a couple of neocortical layers, gives $|q \mathbf{A}| \approx 2 \times 10^{-19} \times 10^{-7} \times$ $10^{-8} / 10^{-4}=10^{-28} \mathrm{~kg}-\mathrm{m} / \mathrm{s}$,

\subsubsection{Reasonable Estimates}

Estimates used here for $\mathbf{Q}$ come from experimental data. These include shielding and material effects. When coherent activity among many macrocolumns associated with STM is considered, $|\mathbf{A}|$ may be much larger. Since $\mathrm{Ca}^{2+}$ waves influence synaptic activity, there is direct coherence between these waves and the activity of $\mathbf{A}$.

Classical physics calculates $q \mathbf{A}$ from macroscopic EEG to be on the order of $10^{-28} \mathrm{~kg}-\mathrm{m} / \mathrm{s}$, while the momentum $\mathbf{p}$ of a $\mathrm{Ca}^{2+}$ ion is on the order of $10^{-30} \mathrm{~kg}-\mathrm{m} / \mathrm{s}$. This numerical comparison demonstrates the possible importance of the influence of $\mathbf{A}$ on $\mathbf{p}$ at classical scales.

This project fits the SMNI model to EEG data. Direct calculations in classical and quantum physics support the concepts presented here, e.g., that ionic calcium momentum-wave effects among neuron-astrocyte-neuron tripartite synapses modify background SMNI parameters and create feedback between ionic/quantum and macroscopic scales (Ingber, 2012a b, 2015, 2016a, 2017a; Ingber et al., 2014; Nunez et al., 2013).

\subsection{Model of Models (MOM)}

Deep Learning (DL) has invigorated AI approaches to parsing data in complex systems, often to develop control processes of these systems. A couple of decades ago, Neural Net AI approaches fell out of favor when concerns were apparent that such approaches offered little guidance to explain the "why" or "how" such algorithms worked to process data, e.g., contexts which were deemed important to deal with future events and outliers, etc.

The success of DL has overshadowed these concerns. However, that should not diminish their importance, especially if such systems are placed in positions to affect lives and human concerns; humans are ultimately responsible for structures they build.

An approach to dealing with these concerns can be called Model of Models (MOM). An argument in favor of MOM is that humans over thousands of years have developed models of reality across many disciplines, e.g., ranging over Physics, Biology, Mathematics, Economics, etc.

A good use of DL might be to process data for a given system in terms of a collection of models, then again use DL to process the models over the same data to determine a superior model of models (MOM). Eventually, large DL (quantum) machines could possess a database of hundreds or 
thousands of models across many disciplines, and directly find the best (hybrid) MOM for a given system.

In particular, SMNI offers a reasonable model upon which to further develop MOM, wherein multiple scales of observed interactions are developed. This is just one example of how physics modeling and computational physics can be used to better understand complex systems.

\subsubsection{Ideas by Statistical Mechanics}

A project sympathetic to this MOM context was proposed as Ideas by Statistical Mechanics (ISM) (Ingber, 2006a, 2007, 2008). using ASA (Ingber, 1989, 1993a, 2012c) to fit parameters of a generic nonlinear multivariate colored-noise Gaussian-Markovian short-time conditional probability distribution to data, useful for many systems.

Models developed using ASA have been applied in many contexts across many systems (Ingber, 1993b), including applications to neural networks (Atiya et al., 2003).

Many of these ASA applications have used Ordinal representations of features, to permit parameterization of their inclusion into models, quite similar in spirit to DL.

ASA can be used again in the expanded context of MOM. This is suggested as a first step in a new discipline to which MOM is to be applied, to help develop a range of parameters useful for DL, as DL by itself may get stuck in non-ideal local minima of the importance-sampled space. Then, after a reasonable range of models is found, DL can take over to permit much more efficient and accurate development of MOM for a given discipline/system.

\section{Adaptive Simulated Annealing (ASA) Algorithm}

\subsection{Importance Sampling}

Nonlinear and/or stochastic systems often require importance-sampling algorithms to scan or to fit parameters. Methods of simulated annealing (SA) are often used. Proper annealing (not "quenching") possesses a proof of finding the deepest minimum in searches.

The ASA code is open-source software, and can be downloaded and used without any cost or registration at https://www.ingber.com/\#ASA (Ingber, 1993a, 2012c).

This algorithm fits empirical data to a cost function over a $D$-dimensional parameter space, adapting for varying sensitivities of parameters during the fit.

This ASA algorithm is faster than fast Cauchy annealing, which has schedule $T_{i}=T_{0} / k$, and much faster than Boltzmann annealing, which has schedule $T_{i}=T_{0} / \ln k$ (Ingber, 1989).

\subsection{Outline of ASA Algorithm}

For parameters

$$
\alpha_{k}^{i} \in\left[A_{i}, B_{i}\right]
$$

sampling with the random variable $x^{i}$

$$
\begin{gathered}
x^{i} \in[-1,1] \\
\alpha_{k+1}^{i}=\alpha_{k}^{i}+x^{i}\left(B_{i}-A_{i}\right)
\end{gathered}
$$

the default generating function is 


$$
g_{T}(x)=\prod_{i=1}^{D} \frac{1}{2 \ln \left(1+1 / T_{i}\right)\left(\left|x^{i}\right|+T_{i}\right)} \equiv \prod_{i=1}^{D} g_{T}^{i}\left(x^{i}\right)
$$

in terms of parameter "temperatures"

$$
T_{i}=T_{i 0} \exp \left(-c_{i} k^{1 / D}\right)
$$

The default ASA uses the same type of annealing schedule for the acceptance function $h$ as used for the generating function $g$.

All default functions in ASA can be overridden with user-defined functions.

\subsection{ASA Applications}

The ASA code (Ingber, 1993a) and the original Very Fast Simulated Reannealing (VFSR) code (Ingber, 1989) have been used by many researchers, including the author in a range of disciplines:

chaotic systems (Ingber et al., 1996)

combat simulations (Ingber, 1993c, 1998a)

financial systems: bonds, equities, futures, options (Ingber, 1990, 1996a, 2000, 2005,

Ingber et al., 2001; Ingber and Mondescu, 2003)

neuroscience (Ingber, 1991, 1992, 1996b, 1997a, 1998b, 2006b, 2009a b, 2012a,b, 2013 ,

2015; Ingber and Nunez, 1995, 2010; Ingber et al., 2014, 1996, Nunez et al., 2013)

optimization per se (Atiya et al., 2003; Ingber, 1989, 1993b, 1996c, 2012c; Ingber and

Rosen, 1992)

\section{Path-Integral Algorithms PATHINT and qPATHINT}

\subsection{Path Integral in Stratonovich (Midpoint) Representation}

The path integral in the Feynman (midpoint) representation is used to examine discretization issues in time-dependent nonlinear systems (Langouche et al., 1979, 1982; Schulman, 1981). (N.b. $g^{\dagger}$ in $D M$ implies a prepoint evaluation.) Unless explicitly stated, the Einstein summation convention is used which implies repeated indices signify summation; bars $|\ldots|$ imply no summation.

$$
\begin{gathered}
P\left[M_{t} \mid M_{t_{0}}\right] d M(t)=\int \ldots \int D M \exp \left(-\min \int_{t_{0}}^{t} d t^{\prime} L\right) \delta\left(M\left(t_{0}\right)=M_{0}\right) \delta\left(M(t)=M_{t}\right) \\
D M=\lim _{u \rightarrow \infty} \prod_{\rho=1}^{u+1} g^{\dagger 1 / 2} \prod_{G}(2 \pi \theta)^{-1 / 2} d M_{\rho}^{G} \\
L\left(\dot{M}^{G}, M^{G}, t\right)=\frac{1}{2}\left(\dot{M}^{G}-h^{G}\right) g_{G G^{\prime}}\left(\dot{M}^{G^{\prime}}-h^{G^{\prime}}\right)+\frac{1}{2} h_{; G}^{G}+R / 6-V \\
\dot{M}^{G}(t) \rightarrow M_{\rho+1}^{G}-M_{\rho}^{G}, M^{G}(t) \rightarrow \frac{1}{2}\left(M_{\rho+1}^{G}+M_{\rho}^{G}\right),[\ldots]_{, G}=\frac{\partial[\ldots]}{\partial M^{G}} \\
h^{G}=g^{G}-\frac{1}{2} g^{-1 / 2}\left(g^{1 / 2} g^{G G^{\prime}}\right)_{, G^{\prime}}, h_{; G}^{G}=h_{, G}^{G}+\Gamma_{G F}^{F} h^{G}=g^{-1 / 2}\left(g^{1 / 2} h^{G}\right)_{, G}
\end{gathered}
$$




$$
\begin{gathered}
g_{G G^{\prime}}=\left(g^{G G^{\prime}}\right)^{-1}, g=\operatorname{det}\left(g_{G G^{\prime}}\right) \\
\Gamma_{J K}^{F} \equiv g^{L F}[J K, L]=g^{L F}\left(g_{J L, K}+g_{K L, J}-g_{J K, L}\right) \\
R=g^{J L} R_{J L}=g^{J L} g^{J K} R_{F J K L} \\
R_{F J K L}=\frac{1}{2}\left(g_{F K, J L}-g_{J K, F L}-g_{F L, J K}+g_{J L, F K}\right)+g_{M N}\left(\Gamma_{F K}^{M} \Gamma_{J L}^{N}-\Gamma_{F L}^{M} \Gamma_{J K}^{N}\right)
\end{gathered}
$$

Non-constant diffusions add terms to drifts, and a Riemannian-curvature potential $R / 6$ is induced for dimension $>1$ in the Stratonovich/Feynman discretization.

\subsection{Path Integral in Ito (Prepoint) Representation}

In the Ito (prepoint) representation:

$$
\begin{gathered}
P\left[M_{t} \mid M_{t_{0}}\right] d M(t)=\int \ldots \int D M \exp \left(-\min \int_{t_{0}}^{t} d t^{\prime} L\right) \delta\left(M\left(t_{0}\right)=M_{0}\right) \delta\left(M(t)=M_{t}\right) \\
D M=\lim _{u \rightarrow \infty} \prod_{\rho=1}^{u+1} g^{1 / 2} \prod_{G}(2 \pi \Delta t)^{-1 / 2} d M_{\rho}^{G} \\
L\left(\dot{M}^{G}, M^{G}, t\right)=\frac{1}{2}\left(\dot{M}^{G}-g^{G}\right) g_{G G^{\prime}}\left(\dot{M}^{G^{\prime}}-g^{G^{\prime}}\right)+R / 6 \\
\dot{M}^{G}(t) \rightarrow M_{\rho+1}^{G}-M_{\rho}^{G}, M^{G}(t) \rightarrow M_{\rho}^{G} \\
\left(g_{G G^{\prime}}\right)=\left(g^{G G^{\prime}}\right)^{-1}, g=\operatorname{det}\left(g_{G G^{\prime}}\right)
\end{gathered}
$$

Here the diagonal diffusions are $g^{|G G|}$ and the drifts are $g^{G}$.

\subsection{Path-Integral Riemannian Geometry}

The midpoint derivation derives a Riemannian geometry with metric defined by the inverse of the covariance matrix

$$
g_{G G^{\prime}}=\left(g^{G G^{\prime}}\right)^{-1}
$$

and where $R$ is the Riemannian curvature

$$
R=g^{J L} R_{J L}=g^{J L} g^{J K} R_{F J K L}
$$

An Ito prepoint discretization for the same probability distribution $P$ gives a simpler algebraic form,

$$
M\left(\bar{t}_{s}\right)=M\left(t_{s}\right)
$$




$$
L=\frac{1}{2}\left(d M^{G} / d t-g^{G}\right) g_{G G^{\prime}}\left(d M^{G^{\prime}} / d t-g^{G^{\prime}}\right)-V
$$

but the Lagrangian $L$ so specified does not satisfy a variational principle useful for moderate to large noise. Its variational principle is only useful in the weak-noise limit. This often means that finer meshes are required.

\subsection{Three Approaches Are Mathematically Equivalent}

Three basic different approaches are mathematically equivalent:

(a) Fokker-Planck/Chapman-Kolmogorov partial-differential equations

(b) Langevin coupled stochastic-differential equations

(c) Lagrangian or Hamiltonian path-integrals

All three are described here as many researchers are familiar with at least one of these approaches to complex systems.

The path-integral approach is useful to define intuitive physical variables from the Lagrangian $L$ in terms of underlying variables $M^{G}$ :

$$
\begin{gathered}
\text { Momentum }: \Pi^{G}=\frac{\partial L}{\partial\left(\partial M^{G} / \partial t\right)} \\
\text { Mass : } g_{G G^{\prime}}=\frac{\partial L}{\partial\left(\partial M^{G} / \partial t\right) \partial\left(\partial M^{G^{\prime}} / \partial t\right)} \\
\text { Force }: \frac{\partial L}{\partial M^{G}} \\
F=m a: \delta L=0=\frac{\partial L}{\partial M^{G}}-\frac{\partial}{\partial t} \frac{\partial L}{\partial\left(\partial M^{G} / \partial t\right)}
\end{gathered}
$$

Differentiation especially of noisy systems often introduces more noise. The path-integral often gives superior numerical performance because integration is a smoothing process.

\subsubsection{Stochastic Differential Equation (SDE)}

The Stratonovich (midpoint discretized) Langevin equations can be analyzed in terms of the Wiener process $d W^{i}$. This can be developed with Gaussian noise $\eta^{i}=d W^{i} / d t$, with some care taken in the limit of small $d t$.

$$
\begin{gathered}
d M^{G}=f^{G}(t, M(t)) d t+\hat{g}_{i}^{G}(t, M(t)) d W^{i} \\
\dot{M}^{G}(t)=f^{G}(t, M(t))+\hat{g}_{i}^{G}(t, M(t)) \eta^{i}(t) \\
d W^{i} \rightarrow \eta^{i} d t \\
M=\left\{M^{G} ; G=1, \ldots, \Lambda\right\} \\
\eta=\left\{\eta^{i} ; i=1, \ldots, N\right\}
\end{gathered}
$$




$$
\begin{gathered}
\dot{M}^{G}=d M^{G} / d t \\
<\eta^{j}(t)>_{\eta}=0,<\eta^{j}(t), \eta^{j^{\prime}}\left(t^{\prime}\right)>_{\eta}=\delta^{j j^{\prime}} \delta\left(t-t^{\prime}\right)
\end{gathered}
$$

$\eta^{i}$ represents Gaussian white noise.

\subsubsection{Partial Differential Equation (PDE)}

The Fokker-Planck, sometimes defines as Chapman-Kolmogorov, partial differential equation is:

$$
\begin{gathered}
P_{, t}=\frac{1}{2}\left(g^{G G^{\prime}} P\right)_{, G G^{\prime}}-\left(g^{G} P\right)_{, G}+V P \\
P=<P_{\eta}>_{\eta} \\
g^{G}=f^{G}+\frac{1}{2} \hat{g}_{i}^{G^{\prime}} \hat{g}_{i, G^{\prime}}^{G} \\
g^{G G^{\prime}}=\hat{g}_{i}^{G} \hat{g}_{i}^{G^{\prime}} \\
(\ldots)_{, G}=\partial(\ldots) / \partial M^{G}
\end{gathered}
$$

$g^{G}$ replaces $f^{G}$ in the SDE if the Ito (prepoint discretized) calculus is used. If boundary conditions are added as Lagrange multipliers, these enter as a "potential" $V$ creating a Schrodinger-type equation.

\subsection{PATHINT Applications}

Path integrals and PATHINT have been applied across several disciplines, including combat simulations (Ingber et al., 1991), neuroscience (Ingber, 1994, 2017b; Ingber and Nunez, 1995, 2010), finance (Ingber, 2000, 2016b, 2017a b c; Ingber et al., 2001; Ingber and Wilson, 2000), and other nonlinear systems (Ingber, 1995c, 1998a; Ingber et al., 1996).

\subsection{PATHINT/qPATHINT Code}

qPATHINT is an N-dimensional code developed to calculate the propagation of quantum variables in the presence of shocks. Many real systems propagate in the presence of sudden changes of state dependent on time. qPATHINT is based on the classical-physics code, PATHINT, which has been useful in several systems across several disciplines. Applications have been made to SMNI and Statistical Mechanics of Financal Markets (SMFM) (Ingber, 2017a b c).

To numerically calculate the path integral for serial changes in time, standard Monte Carlo techniques generally are not useful. PATHINT was originally developed for this purpose. The PATHINT C code of about 7500 lines of code using the GCC C-compiler was rewritten to use double complex variables instead of double variables, and further developed for arbitrary $\mathrm{N}$ dimensions, creating qPATHINT. The outline of the code is described here for classical or quantum systems, using generic coordinates $q$ (Ingber, 2016b, 2017a c).

The distribution (probabilities for classical systems, wave-functions for quantum systems) can be numerically approximated to a high degree of accuracy using a histogram procedure, developing sums of rectangles of height $P_{i}$ and width $\Delta q^{i}$ at points $q^{i}$. 


\subsubsection{Shocks}

Many real-world systems propagate in the presence of continual "shocks".

In SMNI, collisions occur via regenerative $\mathrm{Ca}^{2+}$ waves. There also are interactions with changing A due to changing highly synchronous neuronal firings.

In SMFM applications, shocks occur due to future dividends, changes in interest rates, changes in asset distributions, etc.

\subsubsection{PATHINT/qPATHINT Histograms}

A one-dimensional path-integral in variable $q$ in the prepoint Ito discretization is developed in terms of the kernel/propagator $G$, for each of its intermediate integrals, as

$$
\begin{gathered}
P(q ; t+\Delta t)=\int d q^{\prime}\left[g^{1 / 2}(2 \pi \Delta t)^{-1 / 2} \exp (-L \Delta t)\right] P\left(q^{\prime} ; t\right)=\int d q^{\prime} G\left(q, q^{\prime} ; \Delta t\right) P\left(q^{\prime} ; t\right) \\
P(q ; t)=\sum_{i=1}^{N} \pi\left(q-q^{i}\right) P_{i}(t) \\
\pi\left(q-q^{i}\right)=1,\left(q^{i}-\frac{1}{2} \Delta q^{i-1}\right) \leq q \leq\left(q^{i}+\frac{1}{2} \Delta q^{i}\right) ; 0, \text { otherwise }
\end{gathered}
$$

This yields

$$
\begin{gathered}
P_{i}(t+\Delta t)=T_{i j}(\Delta t) P_{j}(t) \\
T_{i j}(\Delta t)=\frac{2}{\Delta q^{i-1}+\Delta q^{i}} \int_{q^{i}-\Delta q^{i-1 / 2}}^{q^{i}+\Delta q^{i} / 2} d q \int_{q^{j}-\Delta q^{j-1} / 2}^{q^{j}+\Delta q^{j} / 2} d q^{\prime} G\left(q, q^{\prime} ; \Delta t\right)
\end{gathered}
$$

$T_{i j}$ is a banded matrix representing the Gaussian nature of the short-time probability centered about the drift.

Several projects have used this algorithm (Ingber and Nunez, 1995; Ingber et al., 1996; Ingber and Wilson, 1999; Wehner and Wolfer, 1983a, b, 1987). Special 2-dimensional codes were developed for specific projects in Statistical Mechanics of Combat (SMC), SMNI and SMFM (Ingber, 2000, Ingber et al., 1991; Ingber and Nunez, 1995).

\subsubsection{Meshes For [q]PATHINT}

Explicit dependence of $L$ on time $t$ can be included. The mesh $\Delta q^{i}$ is strongly dependent on diagonal elements of the diffusion matrix, e.g.,

$$
\Delta q^{i} \approx\left(\Delta t g^{|i i|}\right)^{1 / 2}
$$

This constrains the dependence of the covariance of each variable to be a (nonlinear) function of that variable to present a rectangular underlying mesh. Since integration is inherently a smoothing process (Ingber, 1990), coarser meshes are used relative to the corresponding stochastic differential equation(s) (Wehner and Wolfer, 1983a).

By considering the contributions to the first and second moments, conditions on the time and variable meshes can be derived. Thus $\Delta t$ can be measured by the diffusion divided by the square of the drift. 


\subsection{Lessons Learned From SMFM and SMNI}

SMNI qPATHINT has emphasized the requirement of broad-banded kernels for oscillatory quantum states.

SMFM PATHTREE, and its derived qPATHTREE, is a different options code, based on pathintegral error analyses, permitting a new very fast binary calculation, also applied to nonlinear timedependent systems (Ingber et al., 2001). However, in contrast to the present PATHINT/qPATHINT code that has been generalized to N dimensions, currently an SMFM [q]PATHTREE is only a binary tree with $J=1$ and cannot be effectively applied to quantum oscillatory systems (Ingber, 2016b, 2017a c).

\subsubsection{Calculations At Each Node At Each Time Slice}

SMFM [q]PATHINT for (American) financial options: Calculate at each node of each time slice back in time.

SMNI [q]PATHINT: Calculate at each node of each time slice - forward in time.

At each node of each time slice, a proposed algorithm is to calculate quantum-scale $\mathrm{Ca}^{2+}$ wavepacket (2-way) interactions with macroscopic large-scale EEG/A. This entails algorithms:

PATHINT using the Classical SMNI Lagrangian qPATHINT using the Quantum $\mathrm{Ca}^{2+}$ wave-packet Lagrangian

Sync in time during P300 attentional tasks.

Time/phase relations between classical and quantum systems may be important. ASA-fit synchronized classical-quantum PATHINT-qPATHINT model to EEG data.

A is determined experimentally from EEG, and includes all synaptic background $B_{G^{\prime}}^{G}$ effects.

\section{$5 \quad$ Results Including Quantum Scales}

The wave function $\psi_{\mathrm{e}}$ describing the interaction of $\mathbf{A}$ with $\mathbf{p}$ of $\mathrm{Ca}^{2+}$ wave packets was derived in closed form from the Feynman representation of the path integral using path-integral techniques (Schulten, 1999), modified here to include A.

$$
\begin{gathered}
\psi_{\mathrm{e}}(t)=\int d \mathbf{r}_{0} \psi_{0} \psi_{F}=\left[\frac{1-i \hbar t /\left(m \Delta \mathbf{r}^{2}\right)}{1+i \hbar t /\left(m \Delta \mathbf{r}^{2}\right)}\right]^{1 / 4}\left[\pi \Delta \mathbf{r}^{2}\left\{1+\left[\hbar t /\left(m \Delta \mathbf{r}^{2}\right)\right]^{2}\right\}\right]^{-1 / 4} \\
\times \exp \left[-\frac{\left[\mathbf{r}-\left(\mathbf{p}_{0}+q \mathbf{A}\right) t / m\right]^{2}}{2 \Delta \mathbf{r}^{2}} \frac{1-i \hbar t /\left(m \Delta \mathbf{r}^{2}\right)}{1+\left[\hbar t /\left(m \Delta \mathbf{r}^{2}\right)\right]^{2}}+i \frac{\mathbf{p}_{0} \cdot \mathbf{r}}{\hbar}-i \frac{\left(\mathbf{p}_{0}+q \mathbf{A}\right)^{2} t}{2 \hbar m}\right] \\
\psi_{F}(t)=\int \frac{d \mathbf{p}}{2 \pi \hbar} \exp \left[\frac{i}{\hbar}\left(\mathbf{p}\left(\mathbf{r}-\mathbf{r}_{0}\right)-\frac{\mathbf{\Pi}^{2} t}{(2 m)}\right)\right]=\left[\frac{m}{2 \pi i \hbar t}\right]^{1 / 2} \exp \left[\frac{i m\left(\mathbf{r}-\mathbf{r}_{0}-q \mathbf{A} t / m\right)^{2}}{2 \hbar t}-\frac{i(q \mathbf{A})^{2} t}{2 m \hbar}\right] \\
\psi_{0}=\psi\left(\mathbf{r}_{0}, t=0\right)=\left(\frac{1}{\pi \Delta \mathbf{r}^{2}}\right)^{1 / 4} \exp \left(-\frac{\mathbf{r}_{0}^{2}}{2 \Delta \mathbf{r}^{2}}+i \frac{\mathbf{p}_{0} \cdot \mathbf{r}_{0}}{\hbar}\right)
\end{gathered}
$$

where $\psi_{0}$ is the initial Gaussian packet, $\psi_{F}$ is the free-wave evolution operator, $\hbar$ is the Planck constant, $q$ is the electronic charge of $\mathrm{Ca}^{2+}$ ions, $m$ is the mass of a wave-packet of $1000 \mathrm{Ca}^{2+}$ ions, $\Delta \mathbf{r}^{2}$ is the spatial variance of the wave-packet, the initial momentum is $\mathbf{p}_{0}$, and the evolving canonical momentum is $\boldsymbol{\Pi}=\mathbf{p}+q \mathbf{A}$. Detailed calculations show that $\mathbf{p}$ of the $\mathrm{Ca}^{2+}$ wave packet and $q \mathbf{A}$ of the EEG field make about equal contributions to $\boldsymbol{\Pi}$ (Ingber, 2015). 


\section{1 $\mathrm{SMNI}+\mathrm{Ca}^{2+}$ wave-packet}

Tripartite influence on synaptic $B_{G^{\prime}}^{G}$ is measured by the ratio of packet's $<\mathbf{p}(t)>_{\psi * \psi}$ to $<$ $\mathbf{p}_{0}\left(t_{0}\right)>_{\psi * \psi}$ at the onset of each attentional task. Here $<>_{\psi * \psi}$ is taken over $\psi_{\mathrm{e}}^{*} \psi_{\mathrm{e}}$.

$$
<\mathbf{p}>_{\psi * \psi}=m \frac{<\mathbf{r}>_{\psi * \psi}}{t-t_{0}}=\frac{q \mathbf{A}+\mathbf{p}_{0}}{m^{1 / 2}|\Delta \mathbf{r}|}\left(\frac{(\hbar t)^{2}+\left(m \Delta \mathbf{r}^{2}\right)^{2}}{\hbar t+m \Delta \mathbf{r}^{2}}\right)^{1 / 2}
$$

A changes slower than $\mathbf{p}$, so static approximation of $\mathbf{A}$ used to derive $\psi_{\mathrm{e}}$ and $\langle\mathbf{p}\rangle_{\psi * \psi}$ is reasonable to use within P300 EEG epochs, resetting $t=0$ at the onset of each classical EEG measurement (1.953 ms apart), using the current A. This permits tests of interactions across scales in a classical context.

\section{$5.2 \quad$ Supercomputer Resources}

The XSEDE.org University of California San Diego (UCSD) supercomputer resource is Comet, described at https://portal.xsede.org/sdsc-comet .

About 1000 hours of supercomputer CPUs are required for an ASA fit of SMNI to the same EEG data used previously, i.e., from http://physionet.nlm.nih.gov/pn4/erpbci (Citi et al., 2010, Goldberger et al., 2000), using mostly the same codes used previously (Ingber, 2016a). Many such sets of runs are required. Including quantum processes will take even longer.

\subsection{Results Using $<\mathbf{p}>_{\psi * \psi}$}

$<\mathbf{p}>_{\psi * \psi}$ was used in classical-physics SMNI fits to EEG data using ASA. Runs using 1M or 100K generated states gave results not much different. Training with ASA used 100K generated states over 12 subjects with and without $\mathbf{A}$, followed by 1000 generated states with the simplex local code contained with ASA. Training and Testing runs on XSEDE.org for this project has taken an equivalent of several months of CPU on the XSEDE.org UCSD platform Comet. These calculations use one additional parameter across all EEG regions to weight the contribution to synaptic background $B_{G^{\prime}}^{G}$. A is taken to be proportional to the currents measured by EEG, i.e., firings $M^{G}$. Otherwise, the "zero-fit-parameter" SMNI philosophy was enforced, wherein parameters are picked from experimentally determined values or within experimentally determined ranges (Ingber, 1984).

As with previous studies using this data, results sometimes give Testing cost functions less than the Training cost functions. This reflects on great differences in data, likely from great differences in subjects' contexts, e.g., possibly due to subjects' STM strategies only sometimes including effects calculated here. Further tests of these multiple-scale models with more EEG data are required, and with the PATHINT-qPATHINT coupled algorithm described previously.

Table 1 gives recent results on such tests. Cost functions are the effective Action, $A_{\text {eff }}$, which is $L \Delta t-\log$ (prefactor), where the prefactor multiplier of the exponential arises from the normalization of the short-time conditional probability distribution and $L \Delta t$ is the argument of the exponential factor. Eq. (3) defines the Lagrangian $L$, and the normalization is defined in $D M$ in Eq. (11).

\subsection{Quantum Zeno Effects}

The quantum-mechanical wave function of the wave packet was shown to "survive" overlaps after multiple collisions, due to their regenerative processes during the observed long durations of hundreds of ms. Thus, $\mathrm{Ca}^{2+}$ waves may support a Zeno or "bang-bang" effect which may promote long coherence times (Burgarth et al., 2018, Facchi et al., 2004, Facchi and Pascazio, 2008, Giacosa 
Table 1: Column 1 is the subject number; the other columns are cost functions. Columns 2 and 3 are no-A model's Training (TR0) and Testing (TE0). Columns 4 and 5 are A model's Training (TRA) and Testing (TEA). Columns 6 and 7 are switched no-A model's Training (sTR0) and Testing (sTE0). Columns 8 and 9 are switched A model's Training (sTRA) and Testing (sTEA).

\begin{tabular}{|l|rr|rr|rr|rr|}
\hline Sub & TR0 & TE0 & TRA & TEA & sTR0 & sTE0 & sTRA & sTEA \\
\hline s01 & 85.75 & 121.23 & 84.76 & 121.47 & 120.48 & 86.59 & 119.23 & 87.06 \\
s02 & 70.80 & 51.21 & 68.63 & 56.51 & 51.10 & 70.79 & 49.36 & 74.53 \\
s03 & 61.37 & 79.81 & 59.83 & 78.79 & 79.20 & 61.50 & 75.22 & 79.17 \\
s04 & 52.25 & 64.20 & 50.09 & 66.99 & 63.55 & 52.83 & 63.27 & 64.60 \\
s05 & 67.28 & 72.04 & 66.53 & 72.78 & 71.38 & 67.83 & 69.60 & 68.13 \\
s06 & 84.57 & 69.72 & 80.22 & 64.13 & 69.09 & 84.67 & 61.74 & 114.21 \\
s07 & 68.66 & 78.65 & 68.28 & 86.13 & 78.48 & 68.73 & 75.57 & 69.58 \\
s08 & 46.58 & 43.81 & 44.24 & 49.38 & 43.28 & 47.27 & 42.89 & 63.09 \\
s09 & 47.22 & 24.88 & 46.90 & 25.77 & 24.68 & 47.49 & 24.32 & 49.94 \\
s10 & 53.18 & 33.33 & 53.33 & 36.97 & 33.14 & 53.85 & 30.32 & 55.78 \\
s11 & 43.98 & 51.10 & 43.29 & 52.76 & 50.95 & 44.47 & 50.25 & 45.85 \\
s12 & 45.78 & 45.14 & 44.38 & 46.08 & 44.92 & 46.00 & 44.45 & 46.56 \\
\hline
\end{tabular}

and Pagliara, 2014; Kozlowski et al., 2015, Muller et al., 2016; Patil et al., 2015; Wu et al., 2012, Zhang et al., 2014).

Of course, the Zeno/"bang-bang" effect may exist only in special contexts, since decoherence among particles is known to be very fast, e.g., faster than phase-damping of macroscopic classical particles colliding with quantum particles (Preskill, 2015).

The wave may be perpetuated by the constant collisions of ions as they enter and leave the wave packet due to the regenerative collisions by the Zeno/ "bang-bang" effect. qPATHINT can calculate the coherence stability of the wave due to serial shocks.

\subsubsection{Survival of Wave Packet}

In momentum space, the wave packet $\phi(\mathbf{p}, t)$ is considered as being "kicked" from $\mathbf{p}$ to $\mathbf{p}+\delta \mathbf{p}$. Assume that random repeated kicks of $\delta \mathbf{p}$ result in $\langle\delta \mathbf{p}\rangle \approx 0$, and that each kick keeps the variance $\Delta(\mathbf{p}+\delta \mathbf{p})^{2} \approx \Delta(\mathbf{p})^{2}$. Then, the overlap integral at the moment $t$ of a typical kick between the new and old state is

$$
\begin{gathered}
<\phi^{*}(\mathbf{p}+\delta \mathbf{p}, t) \mid \phi(\mathbf{p}, t)>=\exp \left(\frac{i \kappa+\rho}{\sigma}\right) \\
\kappa=8 \delta \mathbf{p} \Delta \mathbf{p}^{2} \hbar m\left(q \mathbf{A}+\mathbf{p}_{0}\right) t-4\left(\delta \mathbf{p} \Delta \mathbf{p}^{2} t\right)^{2} \\
\rho=-(\delta \mathbf{p} \hbar m)^{2} \\
\sigma=8(\Delta \mathbf{p} \hbar m)^{2}
\end{gathered}
$$

where $\phi(\mathbf{p}+\delta \mathbf{p}, t)$ is the normalized wave function in $\mathbf{p}+\delta \mathbf{p}$ momentum space. A crude estimate is obtained of the survival time amplitude $A(t)$ and survival probability $p(t)$ (Facchi and Pascazio, 2008), 


$$
\begin{gathered}
A(t)=<\phi^{*}(\mathbf{p}+\delta \mathbf{p}, t) \mid \phi(\mathbf{p}, t)> \\
p(t)=|A(t)|^{2}
\end{gathered}
$$

These numbers yield:

$$
<\phi^{*}(\mathbf{p}+\delta \mathbf{p}, t) \mid \phi(\mathbf{p}, t)>=\exp \left(i\left(1.67 \times 10^{-1} t-1.15 \times 10^{-2} t^{2}\right)-1.25 \times 10^{-7}\right)
$$

Even many small repeated kicks do not appreciably affect the real part of $\phi$, and these projections do not appreciably destroy the original wave packet, giving a survival probability per kick as $p(t) \approx \exp \left(-2.5 \times 10^{-7}\right) \approx 1-2.5 \times 10^{-7}$.

The time-dependent phase terms are sensitive to times of tenths of a sec. These times are prominent in STM and in synchronous neural firings. Therefore, $\mathbf{A}$ effects on $\mathrm{Ca}^{2+}$ wave functions may maximize their influence on STM at frequencies consistent with synchronous EEG during STM.

All these calculations support this model, in contrast to other models of quantum brain processes without such specific calculations and support (Hagan et al., 2002; Hameroff and Penrose, 2013, McKemmish et al., 2009).

\section{Quantum Applications}

\subsection{Nano-Robotic Applications}

There is the possibility of carrying pharmaceutical products in nanosystems that could affect unbuffered $\mathrm{Ca}^{2+}$ waves in neocortex (Ingber, 2015). A Ca ${ }^{2+}$-wave momentum-sensor could act like a piezoelectric device.

At the onset of a $\mathrm{Ca}^{2+}$ wave (on the order of 100's of ms), a change of momentum can be on the order of $10^{-30} \mathrm{~kg}-\mathrm{m} / \mathrm{s}$ for a typical $\mathrm{Ca}^{2+}$ ion. $\mathrm{A} \mathrm{Ca}^{2+}$ wave packet of 1000 ions with onset time of $1 \mathrm{~ms}$, exerts a force on the order of $10^{-24} \mathrm{~N}\left(1 \mathrm{~N} \equiv 1\right.$ Newton $\left.=1 \mathrm{~kg}-\mathrm{m} / \mathrm{s}^{2}\right)$. A nano-robot would be attracted to this site, depositing chemicals/drugs that interact with the regenerative $\mathrm{Ca}^{2+}$-wave process.

An area of the receptor of the nanosystem of $1 \mathrm{~nm}^{2}$ would require pressure sensitivity of $10^{-6} \mathrm{~Pa}$ $\left(1 \mathrm{~Pa}=1\right.$ pascal $\left.=1 \mathrm{~N} / \mathrm{m}^{2}\right)$.

The nano-roboot could be switched on/off at a regional/columnar level by sensitivity to local electric/magnetic fields. Highly synchronous firings during STM processes can be affected by these piezoelectric nanosystems which affect background/noise efficacies via control of $\mathrm{Ca}^{2+}$ waves. In turn, this would affect the influence of of $\mathrm{Ca}^{2+}$ waves via the vector potential $\mathbf{A}$, etc.

\subsection{Free Will}

There is interest in researching possible quantum influences on highly synchronous neuronal firings relevant to STM to understand connections to consciousness and "Free Will" (FW) (Ingber, $2016 \mathrm{a}$ b).

If experimental evidence is gained of quantum-level processes of tripartite synaptic interactions with large-scale synchronous neuronal firings, then FW may be established using the ConwayKochen quantum no-clone "Free Will Theorem" (FWT) (Conway and Kochen, 2006, 2009).

The essence of FWT is that, since quantum states cannot be cloned, a $\mathrm{Ca}^{2+}$ quantum wavepacket may not generate a state proven to have previously existed. As explained by the authors 
(Conway and Kochen, 2006, 2009), experimenters have specific choices in selecting measurements, which are shared by (twinned) particles, including the choice of any random number generator that might be used to aid such choices. The authors maintain that their proof and description of quantum measurements used is general enough to rule out classical randomness, and that classical determinism cannot be supported by such processes as exist in the quantum world.

\section{Conclusion}

The SMNI model has demonstrated it is faithful to experimental data, for EEG recordings under STM experimental paradigms. qPATHINT permits an inclusion of quantum scales in the multiplescale SMNI model, by evolving $\mathrm{Ca}^{2+}$ wave-packets with momentum p, including serial shocks, interacting with the magnetic vector potential A derived from EEG data, marching forward in time lock-step with experimental EEG data. This presents a time-dependent propagation of interacting quantum and classical scales.

This quantum path-integral algorithm with serial random shocks will be further studied as it can be used for many quantum systems.

\section{Acknowledgment}

The author thanks the Extreme Science and Engineering Discovery Environment (XSEDE.org), for supercomputer grants since February 2013, starting with "Electroencephalographic field influence on calcium momentum waves", one under PHY130022 and two under TG-MCB140110. The current grant under TG-MCB140110, "Quantum path-integral qPATHTREE and qPATHINT algorithms", was started in 2017, and renewed through December 2018. XSEDE grants have spanned several projects described in https://www.ingber.com/lir_computational_physics_group.html . 


\section{References}

J. A. Pereira and F. Furlan, "On the role of synchrony for neuron-astrocyte interactions and perceptual conscious processing," Journal of Biological Physics, vol. 35, no. 4, pp. 465-480, 2009 .

C. Agulhon, J. Petravicz, A. McMullen, E. Sweger, S. Minton, S. Taves, K. Casper, T. Fiacco, and K. McCarthy, "What is the role of astrocyte calcium in neurophysiology?" Neuron, vol. 59, pp. 932-946, 2008.

A. Araque and M. Navarrete, "Glial cells in neuronal network function," Philosophical Transactions of The Royal Society B, pp. 2375-2381, 2010.

J. Asher, "Brain's code for visual working memory deciphered in monkeys NIH-funded study," NIH, Bethesda, MD, Tech. Rep. NIH Press Release, 2012, http://www.nimh.nih.gov/news/ science-news/2012/in-sync-brain-waves-hold-memory-of-objects-just-seen.shtml.

A. Atiya, A. Parlos, and L. Ingber, "A reinforcement learning method based on adaptive simulated annealing," in Proceedings International Midwest Symposium on Circuits and Systems (MWCAS), December 2003. Cairo, Egypt: IEEE CAS, 2003, https://www.ingber.com/ asa03_reinforce.pdf

M. Banaclocha, I. Bookkon, and H. Banaclocha, "Long-term memory in brain magnetite," Medical Hypotheses, vol. 74, no. 2, pp. 254-257, 2010.

S. Bellinger, "Modeling calcium wave oscillations in astrocytes," Neurocomputing, vol. 65, no. 66, pp. 843-850, 2005.

F. Briggs, G. Mangun, and W. Usrey, "Attention enhances synaptic efficacy and the signal-to-noise ratio in neural circuits," Nature, vol. 000, pp. 1-5, 2013, https://doi.org/10.1038/nature12276.

D. Burgarth, P. Facchi, H. Nakazato, S. Pascazio, and K. Yuasa, "Quantum zeno dynamics from general quantum operations," Aberystwyth U., Aberystwyth, UK, Tech. Rep. arXiv:1809.09570 [quant-ph], 2018, https://arxiv.org/pdf/1510.04857.pdf.

L. Citi, R. Poli, and C. Cinel, "Documenting, modelling and exploiting P300 amplitude changes due to variable target delays in Donchin's speller," Journal of Neural Engineering, vol. 7, no. 056006, pp. 1-21, 2010, https://doi.org/10.1088/1741-2560/7/5/056006.

J. Conway and S. Kochen, "The free will theorem," Princeton U, Princeton, NJ, Tech. Rep. arXiv:quant-ph/0604079 [quant-ph], 2006, https://arxiv.org/pdf/quant-ph/0604079.pdf.

J. Conway and S. Kochen, "The strong free will theorem," Notices of the American Mathematical Society, vol. 56, no. 2, pp. 226-232, 2009.

K. Ericsson and W. Chase, "Exceptional memory," American Scientist, vol. 70, pp. 607-615, 1982.

P. Facchi and S. Pascazio, "Quantum zeno dynamics: mathematical and physical aspects," Journal of Physics A, vol. 41, no. 493001, pp. 1-45, 2008. 
P. Facchi, D. Lidar, and S. Pascazio, "Unification of dynamical decoupling and the quantum zeno effect," Physical Review A, vol. 69, no. 032314, pp. 1-6, 2004.

G. Giacosa and G. Pagliara, "Quantum zeno effect by general measurements," Physical Review A, vol. 052107, pp. 1-5, 2014.

A. Goldberger, L. Amaral, L. Glass, J. Hausdorff, P. Ivanov, R. Mark, J. Mietus, G. Moody, C.K. Peng, and H. Stanley, "PhysioBank, PhysioToolkit, and PhysioNet: components of a new research resource for complex physiologic signals," Circulation, vol. 101, no. 23, pp. e215-e220, 2000, http://circ.ahajournals.org/cgi/content/full/101/23/e215.

S. Hagan, S. Hameroff, and J. Tuszynski, "Quantum computation in brain microtubules: Decoherence and biological feasibility," Physical Review E, vol. 65, no. 061901, pp. 1-11, 2002, https://doi.org/10.1103/PhysRevE.65.061901.

S. Hameroff and R. Penrose, "Consciousness in the universe: A review of the 'Orch OR' theory," Physics of Life Reviews, vol. 403, pp. 1-40, 2013, https://doi.org/10.1016/j.plrev.2013.08.002.

W. Hick, "On the rate of gains of information," Quarterly Journal Experimental Psychology, vol. 34, no. 4, pp. 1-33, 1952.

L. Ingber, "Towards a unified brain theory," Journal Social Biological Structures, vol. 4, pp. 211224, 1981, https://www.ingber.com/smni81_unified.pdf.

L. Ingber, "Statistical mechanics of neocortical interactions. i. basic formulation," Physica D, vol. 5, pp. 83-107, 1982, https://www.ingber.com/smni82_basic.pdf

L. Ingber, "Statistical mechanics of neocortical interactions. dynamics of synaptic modification," Physical Review A, vol. 28, pp. 395-416, 1983, https://www.ingber.com/smni83_dynamics.pdf.

L. Ingber, "Statistical mechanics of neocortical interactions. derivation of short-term-memory capacity," Physical Review A, vol. 29, pp. 3346-3358, 1984, https://www.ingber.com/smni84_stm. pdf.

L. Ingber, "Statistical mechanics of neocortical interactions: Stability and duration of the 7+-2 rule of short-term-memory capacity," Physical Review A, vol. 31, pp. 1183-1186, 1985a, https: //www.ingber.com/smni85_stm.pdf.

L. Ingber, "Towards clinical applications of statistical mechanics of neocortical interactions," Innovations Technology Biology Medicine, vol. 6, pp. 753-758, 1985b.

L. Ingber, "Statistical mechanics of neocortical interactions. EEG dispersion relations," IEEE Transactions in Biomedical Engineering, vol. 32, pp. 91-94, 1985c, https://www.ingber.com/ smni85_eeg.pdf.

L. Ingber, "Very fast simulated re-annealing," Mathematical Computer Modelling, vol. 12, no. 8, pp. 967-973, 1989, https://www.ingber.com/asa89_vfsr.pdf.

L. Ingber, "Statistical mechanical aids to calculating term structure models," Physical Review A, vol. 42, no. 12, pp. 7057-7064, 1990, https://www.ingber.com/markets90_interest.pdf.

L. Ingber, "Statistical mechanics of neocortical interactions: A scaling paradigm applied to electroencephalography," Physical Review A, vol. 44, no. 6, pp. 4017-4060, 1991, https://www. ingber.com/smni91_eeg.pdf. 
L. Ingber, "Generic mesoscopic neural networks based on statistical mechanics of neocortical interactions," Physical Review A, vol. 45, no. 4, pp. R2183-R2186, 1992, https://www.ingber.com/ smni92_mnn.pdf.

L. Ingber, "Adaptive simulated annealing (ASA)," Caltech Alumni Association, Pasadena, CA, Tech. Rep. Global optimization C-code, 1993a, https://www.ingber.com/\#ASA-CODE.

L. Ingber, "Simulated annealing: Practice versus theory," Mathematical Computer Modelling, vol. 18, no. 11, pp. 29-57, 1993b, https://www.ingber.com/asa93_sapvt.pdf.

L. Ingber, "Statistical mechanics of combat and extensions," in Toward a Science of Command, Control, and Communications, C. Jones, Ed. Washington, D.C.: American Institute of Aeronautics and Astronautics, 1993c, pp. 117-149, ISBN 1-56347-068-3. https://www.ingber.com/ combat93_c3sci.pdf.

L. Ingber, "Statistical mechanics of neocortical interactions: Path-integral evolution of short-term memory," Physical Review E, vol. 49, no. 5B, pp. 4652-4664, 1994, https://www.ingber.com/ smni94_stm.pdf.

L. Ingber, "Statistical mechanics of multiple scales of neocortical interactions," in Neocortical Dynamics and Human EEG Rhythms, P. Nunez, Ed. New York, NY: Oxford University Press, 1995a, pp. 628-681, ISBN 0-19-505728-7. https://www.ingber.com/smni95_scales.pdf.

L. Ingber, "Statistical mechanics of neocortical interactions: Constraints on 40 hz models of shortterm memory," Physical Review E, vol. 52, no. 4, pp. 4561-4563, 1995b, https://www.ingber. com/smni95_stm40hz.pdf.

L. Ingber, "Path-integral evolution of multivariate systems with moderate noise," Physical Review E, vol. 51, no. 2, pp. 1616-1619, 1995c, https://www.ingber.com/path95_nonl.pdf.

L. Ingber, "Canonical momenta indicators of financial markets and neocortical EEG," in Progress in Neural Information Processing, S.-I. Amari, L. Xu, I. King, and K.-S. Leung, Eds. New York: Springer, 1996a, pp. 777-784, Invited paper to the 1996 International Conference on Neural Information Processing (ICONIP'96), Hong Kong, 24-27 September 1996. ISBN 981-3083-05-0. https://www.ingber.com/markets96_momenta.pdf

L. Ingber, "Statistical mechanics of neocortical interactions: Multiple scales of EEG," in Frontier Science in EEG: Continuous Waveform Analysis (Electroencephal. clin. Neurophysiol. Suppl. 45), R. Dasheiff and D. Vincent, Eds. Amsterdam: Elsevier, 1996b, pp. 79-112, Invited talk to Frontier Science in EEG Symposium, New Orleans, 9 Oct 1993. ISBN 0-444-82429-4. https: //www.ingber.com/smni96_eeg.pdf.

L. Ingber, "Adaptive simulated annealing (ASA): lessons learned," Control and Cybernetics, vol. 25, no. 1, pp. 33-54, 1996c, Invited paper to Control and Cybernetics on Simulated Annealing Applied to Combinatorial Optimization. https://www.ingber.com/asa96_lessons.pdf.

L. Ingber, "Statistical mechanics of neocortical interactions: Applications of canonical momenta indicators to electroencephalography," Physical Review E, vol. 55, no. 4, pp. 4578-4593, 1997a, https://www.ingber.com/smni97_cmi.pdf.

L. Ingber, EEG Database. Irvine, CA: UCI Machine Learning Repository, 1997b, http://archive. ics.uci.edu/ml/datasets/EEG+Database 
L. Ingber, "Data mining and knowledge discovery via statistical mechanics in nonlinear stochastic systems," Mathematical Computer Modelling, vol. 27, no. 3, pp. 9-31, 1998a, https://www.ingber. com/path98_datamining.pdf.

L. Ingber, "Statistical mechanics of neocortical interactions: Training and testing canonical momenta indicators of EEG," Mathematical Computer Modelling, vol. 27, no. 3, pp. 33-64, 1998b, https://www.ingber.com/smni98_cmi_test.pdf.

L. Ingber, "Statistical mechanics of neocortical interactions: Reaction time correlates of the g factor," Psycholoquy, vol. 10, no. 068, 1999, Invited commentary on The g Factor: The Science of Mental Ability by Arthur Jensen. http://www.cogsci.ecs.soton.ac.uk/cgi/psyc/newpsy?10.068

L. Ingber, "High-resolution path-integral development of financial options," Physica A, vol. 283, no. 3-4, pp. 529-558, 2000, https://www.ingber.com/markets00_highres.pdf.

L. Ingber, "Trading in risk dimensions (TRD)," Lester Ingber Research, Ashland, OR, Tech. Rep. Report 2005:TRD, 2005, https://www.ingber.com/markets05_trd.pdf.

L. Ingber, "Ideas by statistical mechanics (ISM)," Lester Ingber Research, Ashland, OR, Tech. Rep. Report 2006:ISM, 2006a, https://www.ingber.com/smni06_ism.pdf.

L. Ingber, "Statistical mechanics of neocortical interactions: Portfolio of physiological indicators," Lester Ingber Research, Ashland, OR, Tech. Rep. Report 2006:PPI, 2006b, https://www.ingber. com/smni06_ppi.pdf.

L. Ingber, "Ideas by statistical mechanics (ISM)," Journal Integrated Systems Design and Process Science, vol. 11, no. 3, pp. 31-54, 2007, Special Issue: Biologically Inspired Computing.

L. Ingber, "AI and ideas by statistical mechanics (ISM)," in Encyclopedia of Artificial Intelligence, J. Rabunal, J. Dorado, and A. Pazos, Eds. New York: Information Science Reference, 2008, pp. 58-64, ISBN 978-1-59904-849-9.

L. Ingber, "Statistical mechanics of neocortical interactions: Portfolio of physiological indicators," The Open Cybernetics Systemics Journal, vol. 3, no. 14, pp. 13-26, 2009a, https://doi.org/10. 2174/1874110x00903010013.

L. Ingber, "Statistical mechanics of neocortical interactions: Nonlinear columnar electroencephalography," NeuroQuantology Journal, vol. 7, no. 4, pp. 500-529, 2009b, https://www.ingber.com/ smni09_nonlin_column_eeg.pdf.

L. Ingber, "Computational algorithms derived from multiple scales of neocortical processing," in Pointing at Boundaries: Integrating Computation and Cognition on Biological Grounds, J. A. Pereira, E. Massad, and N. Bobbitt, Eds. New York: Springer, 2011, pp. 1-13, Invited Paper. https://doi.org/10.1007/s12559-011-9105-4.

L. Ingber, "Columnar EEG magnetic influences on molecular development of short-term memory," in Short-Term Memory: New Research, G. Kalivas and S. Petralia, Eds. Hauppauge, NY: Nova, 2012a, pp. 37-72, Invited Paper. https://www.ingber.com/smni11_stm_scales.pdf.

L. Ingber, "Influence of macrocolumnar EEG on ca waves," Current Progress Journal, vol. 1, no. 1, pp. 4-8, 2012b, https://www.ingber.com/smni12_vectpot.pdf. 
L. Ingber, "Adaptive simulated annealing," in Stochastic global optimization and its applications with fuzzy adaptive simulated annealing, J. H.A. Oliveira, A. Petraglia, L. Ingber, M. Machado, and M. Petraglia, Eds. New York: Springer, 2012c, pp. 33-61, Invited Paper. https://www. ingber.com/asa11_options.pdf.

L. Ingber, "Electroencephalographic (EEG) influence on Ca2+ waves: Lecture plates," Lester Ingber Research, Ashland, OR, Tech. Rep. Report 2013:LEFI, 2013, 2nd World Neuroscience Online Conference 18 June 2013. https://www.ingber.com/smni13_eeg_ca_lect.pdf.

L. Ingber, "Calculating consciousness correlates at multiple scales of neocortical interactions," in Horizons in Neuroscience Research, A. Costa and E. Villalba, Eds. Hauppauge, NY: Nova, 2015, pp. 153-186, ISBN: 978-1-63482-632-7. Invited paper. https://www.ingber.com/smni15_ calc_conscious.pdf.

L. Ingber, "Statistical mechanics of neocortical interactions: Large-scale EEG influences on molecular processes," Journal of Theoretical Biology, vol. 395, pp. 144-152, 2016a, https://doi.org/10. 1016/j.jtbi.2016.02.003

L. Ingber, "Path-integral quantum PATHTREE and PATHINT algorithms," International Journal of Innovative Research in Information Security, vol. 3, no. 5, pp. 1-15, 2016b, https://www. ingber.com/path16_quantum_path.pdf.

L. Ingber, "Evolution of regenerative ca-ion wave-packet in neuronal-firing fields: Quantum pathintegral with serial shocks," International Journal of Innovative Research in Information Security, vol. 4, no. 2, pp. 14-22, 2017a, https://www.ingber.com/path17_quantum_pathint_shocks. pdf.

L. Ingber, "Quantum path-integral qPATHINT algorithm," The Open Cybernetics Systemics Journal, vol. 11, pp. 119-133, 2017b, https://doi.org/10.2174/1874110X01711010119.

L. Ingber, "Options on quantum money: Quantum path-integral with serial shocks," International Journal of Innovative Research in Information Security, vol. 4, no. 2, pp. 7-13, 2017c, https: //www.ingber.com/path17_quantum_options_shocks.pdf.

L. Ingber and R. Mondescu, "Automated internet trading based on optimized physics models of markets," in Intelligent Internet-Based Information Processing Systems, R. Howlett, N. Ichalkaranje, L. Jain, and G. Tonfoni, Eds. Singapore: World Scientific, 2003, pp. 305-356, Invited paper. https://www.ingber.com/markets03_automated.pdf.

L. Ingber and P. Nunez, "Statistical mechanics of neocortical interactions: High resolution pathintegral calculation of short-term memory," Physical Review E, vol. 51, no. 5, pp. 5074-5083, 1995, https://www.ingber.com/smni95_stm.pdf.

L. Ingber, "Neocortical dynamics at multiple scales: EEG standing waves, statistical mechanics, and physical analogs," Mathematical Biosciences, vol. 229, pp. 160-173, 2010, https://www. ingber.com/smni10_multiple_scales.pdf.

L. Ingber and B. Rosen, "Genetic algorithms and very fast simulated reannealing: A comparison," Mathematical Computer Modelling, vol. 16, no. 11, pp. 87-100, 1992, https://www.ingber.com/ asa92_saga.pdf.

L. Ingber and J. Wilson, "Volatility of volatility of financial markets," Mathematical Computer Modelling, vol. 29, no. 5, pp. 39-57, 1999, https://www.ingber.com/markets99_vol.pdf. 
L. Ingber, "Statistical mechanics of financial markets: Exponential modifications to black-scholes," Mathematical Computer Modelling, vol. 31, no. 8/9, pp. 167-192, 2000, https://www.ingber. com/markets00_exp.pdf.

L. Ingber, H. Fujio, and M. Wehner, "Mathematical comparison of combat computer models to exercise data," Mathematical Computer Modelling, vol. 15, no. 1, pp. 65-90, 1991, https://www. ingber.com/combat91_data.pdf.

L. Ingber, R. Srinivasan, and P. Nunez, "Path-integral evolution of chaos embedded in noise: Duffing neocortical analog," Mathematical Computer Modelling, vol. 23, no. 3, pp. 43-53, 1996, https://www.ingber.com/path96_duffing.pdf.

L. Ingber, C. Chen, R. Mondescu, D. Muzzall, and M. Renedo, "Probability tree algorithm for general diffusion processes," Physical Review E, vol. 64, no. 5, pp. $056702-056707,2001$, https: //www.ingber.com/path01_pathtree.pdf.

L. Ingber, M. Pappalepore, and R. Stesiak, "Electroencephalographic field influence on calcium momentum waves," Journal of Theoretical Biology, vol. 343, pp. 138-153, 2014, https://doi.org/ 10.1016/j.jtbi.2013.11.002

B. Innocenti, V. Parpura, and P. Haydon, "Imaging extracellular waves of glutamate during calcium signaling in cultured astrocytes," Journal of Neuroscience, vol. 20, no. 5, pp. 1800-1808, 2000.

A. Jensen, "Individual differences in the hick paradigm," in Speed of Information-Processing and Intelligence, P. Vernon, Ed. Norwood, NJ: Ablex, 1987, pp. 101-175.

W. Kozlowski, S. Caballero-Benitez, and I. Mekhov, "Non-hermitian dynamics in the quantum zeno limit," U Oxford, Oxford, UK, Tech. Rep. arXiv:1510.04857 [quant-ph], 2015, https://arxiv.org/ pdf/1510.04857.pdf.

F. Langouche, D. Roekaerts, and E. Tirapegui, "Discretization problems of functional integrals in phase space," Physical Review D, vol. 20, pp. 419-432, 1979.

L. Ingber, Functional Integration and Semiclassical Expansions. Dordrecht, The Netherlands: Reidel, 1982.

S. Majhi and D. Ghosh, "Alternating chimeras in networks of ephaptically coupled bursting neurons," Chaos, vol. 28, no. 083113, 2018, https://doi.org/10.1063/1.5022612.

L. McKemmish, J. Reimers, R. McKenzie, A. Mark, and N. Hush, "Penrose-hameroff orchestrated objective-reduction proposal for human consciousness is not biologically feasible," Physical Review E, vol. 80, no. 021912, pp. 1-6, 2009, https://doi.org/10.1103/PhysRevE.80.021912.

V. Mountcastle, R. Andersen, and B. Motter, "The influence of attentive fixation upon the excitability of the light-sensitive neurons of the posterior parietal cortex," Journal of Neuroscience, vol. 1, pp. 1218-1235, 1981.

M. Muller, S. Gherardini, and F. Caruso, "Quantum zeno dynamics through stochastic protocols," U Florence, Florence, Italy, Tech. Rep. arXiv:1607.08871 [quant-ph], 2016, https://arxiv.org/ pdf/1607.08871.pdf.

S. Murakami and Y. Okada, "Contributions of principal neocortical neurons to magnetoencephalography and electroencephalography signals," Journal of Physiology, vol. 575, no. 3, pp. 925-936, 2006. 
P. Nunez and R. Srinivasan, Electric Fields of the Brain: The Neurophysics of EEG, 2nd Ed. London: Oxford University Press, 2006.

P. Nunez, R. Srinivasan, and L. Ingber, "Theoretical and experimental electrophysiology in human neocortex: Multiscale correlates of conscious experience," in Multiscale Analysis and Nonlinear Dynamics: From genes to the brain, M. Pesenson, Ed. New York: Wiley, 2013, pp. 149-178, https://doi.org/10.1002/9783527671632.ch06.

Y. Patil, S. Chakram, and M. Vengalattore, "Measurement-induced localization of an ultracold lattice gas," Physical Review Letters, vol. 115, no. 140402, pp. 1-5, 2015, https://doi.org/10. 1103/PhysRevLett.115.140402.

J. Preskill, "Quantum mechanics," Caltech, Pasadena, CA, Tech. Rep. Lecture Notes, 2015, http://www.theory.caltech.edu/people/preskill/ph219/.

R. Reyes and V. Parpura, "The trinity of Ca2+ sources for the exocytotic glutamate release from astrocytes," Neurochemistry International, vol. 55, no. 3, pp. 1-14, 2009.

W. Ross, "Understanding calcium waves and sparks in central neurons," Nature Reviews Neuroscience, vol. 13, pp. 157-168, 2012.

R. Salazar, N. Dotson, S. Bressler, and C. Gray, "Content-specific fronto-parietal synchronization during visual working memory," Science, vol. 338, no. 6110, pp. 1097-1100, 2012, https://doi. org/10.1126/science.1224000.

E. Scemes and C. Giaume, "Astrocyte calcium waves: What they are and what they do," Glia, vol. 54, no. 7, pp. 716-725, 2006, https://doi.org/10.1002/glia.20374.

L. Schulman, Techniques and Applications of Path Integration. New York: J. Wiley Sons, 1981.

K. Schulten, "Quantum mechanics," U. Illinois, Urbana, IL, Tech. Rep. PHYS480 Lecture Notes, Chapter 2, 1999, http://www.ks.uiuc.edu/Services/Class/PHYS480/.

A. Volterra, N. Liaudet, and I. Savtchouk, "Astrocyte Ca2+ signalling: an unexpected complexity," Nature Reviews Neuroscience, vol. 15, pp. 327-335, 2014.

M. Wehner and W. Wolfer, "Numerical evaluation of path-integral solutions to fokker-planck equations. I," Physical Review A, vol. 27, pp. 2663-2670, 1983a.

M. Wehner and W. Wolfer, "Numerical evaluation of path-integral solutions to fokker-planck equations. II. restricted stochastic processes," Physical Review A, vol. 28, pp. 3003-3011, 1983b.

M. Wehner and W. Wolfer, "Numerical evaluation of path integral solutions to fokker-planck equations. III. time and functionally dependent coefficients," Physical Review A, vol. 35, pp. 17951801, 1987.

S. Wu, L. Wang, and X. Yi, "Time-dependent decoherence-free subspace," Journal of Physics A, vol. 405305, pp. 1-11, 2012.

G. Zhang and H. Simon, "STM capacity for chinese words and idioms: Chunking and acoustical loop hypotheses," Memory Cognition, vol. 13, pp. 193-201, 1985.

P. Zhang, Q. Ai, Y. Li, D. Xu, and C. Sun, "Dynamics of quantum zeno and anti-zeno effects in an open system," Science China Physics, Mechanics Astronomy, vol. 57, no. 2, pp. 194-207, 2014, https://doi.org/10.1007/s11433-013-5377-x. 